IJADR

International Journal of Alcohol and Drug Research

The Official Journal of the Kettil Bruun Society for Social and Epidemiological Research on Alcohol

\title{
“Doc, I didn’t know I was pregnant. Does my baby have fetal alcohol spectrum disorder?”
}

\section{Commentary}

\begin{abstract}
How much alcohol is "safe" during pregnancy? This is a question for which there is no conclusive answer. Although no mother wants to intentionally harm her baby, the reality is that consuming alcohol during pregnancy can lead to a variety of complex developmental disabilities, collectively termed Fetal Alcohol Spectrum Disorder (FASD). The precise dosimetry data for the timing and dose of alcohol in pregnancy is unknown, but several features of alcohol metabolism are well characterized and have significant implications for alcohol's deleterious effects on the developing fetus. There are a number of critical periods in embryonic development, which can be brief or prolonged and that correspond to specific tissues and structures (organ systems). Alcohol at any one of these critical periods can have significant downstream effects on typical fetal development. Alcohol teratogenicity does not produce a single abnormality, but instead can lead to a cluster of birth defects and/or neurodevelopmental abnormalities (i.e., FASD). Each of these cognitive or behavioral problems can vary in its expression, from severe and obvious early in life, to subtle and apparent only years after birth. Based on the variability among women and their fetuses, genetics and environmental influences, it is very unlikely that "absolute risk" for alcohol and pregnancy will ever be established. The age-old question, "How much is too much?", will also remain unanswered. The best advice: "NO exposure equals NO risk."
\end{abstract}

Keywords: fetal alcohol spectrum disorder (FASD), prenatal alcohol exposure (PAE), pregnancy, dose, response

One of the most unanswered and unanswerable questions in the field of reproductive health and antenatal care is, "How much alcohol will hurt my baby?" How much alcohol is "safe" during pregnancy? Although the question has been evaluated extensively in both the human and animal literature, the answer is not simple.

No mother wants to intentionally harm her baby. However, the reality is that consuming alcohol during pregnancy can lead to a variety of complex developmental disabilities, collectively termed fetal alcohol spectrum disorder (FASD). The disabilities range from mild to severe cognitive, behavioral and psychological problems that are persistent and can be highly debilitating. Who would want this for a child? Nobody.

Typically, when studying the effects of drugs for treatment or environmental agents as toxicants, the evaluation involves defining the "dose-response" relationship, which considers 1) the amount of the compound (dose) received and 2) the result (response). However, this relationship in the context of alcohol and FASD is extremely difficult to solve because both parts of the equation are complex and subject to endogenous (i.e., genetics) and exogeneous (i.e., ethnicity) influences that make it near impossible to determine. And this is coming from the experts in the field! No wonder there is confusion.

Generally, people do not know exactly how much alcohol they consume. Liquor and wine are poured into glasses of varying sizes, refilled before they are emptied, and not often measured specifically for quantity. Most people who drink alcohol regularly do not drink the exact same amount on different days, nor would they remember from week to week how much they drank. This makes it extremely difficult to determine the precise amount of alcohol consumed in pregnancy when patients are asked weeks or months after the fact (Jacobson, Chiodo, Sokol, \& Jacobson, 2002). To further complicate the issue, selfreport data from women related to their alcohol consumption during pregnancy can be unreliable in some situations and for many reasons (Morrow-Tlucak, Ernhart, Sokol, Martier, \& Ager, 1989).

\section{Dose}

After alcohol is consumed, it is readily absorbed into the bloodstream and distributes throughout the total body water (vascular component) of the mother. This is a relatively fast process. Alcohol then passes freely through the placenta from the mother's bloodstream into that of the fetus. The fetus may experience a higher dose of alcohol than the mother, because it has little to no ability to metabolize alcohol. Instead of being metabolized, the alcohol accumulates in the amniotic fluid-where the fetus is exposed to it again-before it passes back across the placenta for metabolism and excretion by the mother. This also means that the fetus may be exposed to a higher dose of alcohol for a longer period of time than the mother. The elimination of alcohol by the mother depends on her 
metabolism, which can be affected by other factors such as nutrition, genetics and body weight (Chudley, 2011; Paintner, Williams, \& Burd, 2012a).

The precise dosimetry data for the timing and dose of alcohol in pregnancy is unknown, but several features of alcohol metabolism are well characterized and have significant implications for the developing fetus. The typical blood alcohol concentration for prenatal alcohol exposure is assessed using body weight and frequency of drinking (Paintner et al., 2012a). Persistent alcohol use increases tolerance, which can result in very high blood alcohol concentrations for some individuals. Another key factor is the effect of drinking on consecutive days, when the second exposure episode begins before the blood alcohol concentration from the previous drinking episode has reached zero. In these situations, a higher blood alcohol concentration for the second episode will result, leading to an extended period of exposure for the fetus.

Because alcohol readily absorbs into the bloodstream after consumption and freely passes through the placenta to the fetal compartment, the fetus reaches the same blood alcohol concentration as the mother within minutes. However, the level at which prenatal alcohol damage can be routinely observed based on peak blood alcohol concentration, number of episodes of exposure, total area under the exposure curve over pregnancy, or number of standard drinks across pregnancy (cumulative exposure) remains unknown (Paintner et al., 2012a). The consumption of five drinks over a two-hour period once a week for a woman weighing 130 pounds (i.e., blood alcohol concentration of 0.16) has been proposed as an important threshold for adverse outcomes (Abel, 1998). Currently, the pregnancy exposure data that is relevant to the severity of symptoms in offspring include 1) level of drinking before pregnancy (since about $50 \%$ of pregnancies are unplanned, this results in early gestational exposure, often to binge episodes of drinking); 2) number of binge episodes; 3) number of standard drinks during pregnancy (a standard drink is defined as $14 \mathrm{~g}$ of ethanol); 4) number of days exposed (an increase in days of exposure increases the number of key gestational periods of risk where exposure occurs); and 5) total area under the exposure curve during pregnancy (Paintner et al., 2012a).

The timing of prenatal alcohol exposure during fetal development is also an important issue. There are a number of critical periods in embryonic development, which can be brief or prolonged and that correspond to specific tissues and structures (organ systems). Alcohol at any one of these critical periods can have significant downstream effects on typical fetal development. Data from animal studies and some research in humans demonstrate that organs and tissues that are developing at the time of alcohol exposure are particularly susceptible to the teratogenic effects of alcohol (Clarren, 1986; Michaelis, 1990; Sulik, Johnston, \& Webb, 1981).

Alcohol-induced damage, however, can occur through many different mechanisms (Abel \& Hannigan, 1995) at different times during pregnancy. This means that a dose of alcohol at one point during pregnancy can be significantly more harmful than the same dose at another time. Unfortunately, most of these critical periods in gestation have not been established definitively. Even if they were, most women do not know the exact day or hour of conception and there are slight variations in the timing of fetal development.

Beyond factors related to amount, frequency and timing of maternal alcohol consumption, recent research developments suggest that fetuses may differ in their genetic susceptibility to the effects of prenatal alcohol exposure. Twin studies demonstrate that identical twins seem to be similarly damaged by alcohol while fraternal twins may have very different results, where one twin is more severely affected than the other (Chudley, 2011).

\section{Response}

While the dose is difficult to precisely gauge, so too is the response. Alcohol teratogenicity does not produce a single abnormality; rather, it can lead to a cluster of birth defects and/or neurodevelopmental abnormalities (i.e., FASD). Each of these cognitive or behavioral problems can vary in its expression, from severe and obvious early in life, to subtle and apparent only years after birth. While many studies have focused on children with FASD, others have involved adults with prenatal alcohol exposure, to try to identify the associated long-term neurodevelopmental abnormalities.

While the search to characterize the FASD phenotype continues (Kodituwakku, 2007, 2009; Nash, Sheard, Rovet, \& Koren, 2008; Nash et al., 2013), contributing factors have emerged from extensive neurocognitive and behavioral assessments. Because prenatal alcohol exposure can affect brain development via numerous pathways and at all stages from neurogenesis to myelination, there are a number of diverse outcomes that can result. Neuropsychological functions such as spatial processing (Mattson et al., 2010), concept formation and conceptual set shifting (McGee, Schonfeld, Roebuck-Spencer, Riley, \& Mattson, 2008), memory (Green et al., 2009; Pei, Job, Kully-Martens, \& Rasmussen, 2011), sensory modulation (Atchison, 2007), attention (Lee, Mattson, \& Riley, 2004) and social skills (Kully-Martens, Denys, Treit, Tamana, \& Rasmussen, 2012) are all sensitive to prenatal alcohol exposure; mental health problems are also commonly associated with an FASD diagnosis (Fryer, McGee, Matt, Riley, \& Mattson, 2007; Pei, Denys, Hughes, \& Rasmussen, 2011). These types of changes in brain and behavior resulting from prenatal alcohol exposure have been reviewed extensively in the FASD literature (Kodituwakku, 2009; Malone \& Koren, 2012; Nash et al., 2008; Paintner, Williams, \& Burd, 2012b; Riley, Infante, \& Warren, 2011). Differences in sensory processing abilities, adaptive behavior and neurocognitive function in children with partial FAS (pFAS), alcohol-related neurodevelopmental disorder (ARND) and prenatal exposure to alcohol (PEA) have been compared and contrasted (Carr, Agnihotri, \& Keightley, 2010). Regardless of the diagnosis, a broad range of functional difficulties were observed underscoring the need to conduct a range of 
standardized neuropsychological tests that will reveal individual patterns of cognitive dysfunction. Recently, there has been a shift in the approach to identifying a neurocognitive profile for children with prenatal alcohol exposure (PAE) (Quattlebaum \& O'Connor, 2012). Instead of a specific profile, current views favour a "generalized deficit" conceptualization marked by diffuse information processing and integration difficulties. After undergoing a comprehensive, multi-informant assessment of neurocognitive, emotional, social, behavioral, and adaptive functioning, and, not surprisingly, children with FASD performed considerably more poorly than the non-exposed control group. Significantly, no differences were found across FASD subtypes, providing evidence to support the emerging generalized deficit conceptualization theory for PEA. Despite these and other findings that have contributed to the field, no one study to date has followed alcohol-exposed children over the lifetime with sufficient objective tests to truly establish the relationship between any dose or pattern of alcohol consumption and individual outcomes.

\section{“Doc, I didn't know I was pregnant. Does my baby have fetal alcohol spectrum disorder?”}

To get back to where we started, how should "we” manage women of childbearing age around issues of prenatal exposure to alcohol? What are the recommendations? What do we know conclusively? Based on the variability among women and their fetuses, genetics and environmental influences, it is very unlikely that "absolute risk" for alcohol and pregnancy will ever be established. The age-old question, "How much is too much?”, will also remain unanswered.

The best advice: "NO exposure equals NO risk.”

\section{References}

Abel, E. L. (1998). Fetal Alcohol Abuse Syndrome. New York, NY: Plenum Press.

Abel, E. L., \& Hannigan, J. H. (1995). Maternal risk factors in fetal alcohol syndrome: Provocative and permissive influences. Neurotoxicology and Teratology, 17, 445462.

Atchison, B. J. (2007). Sensory modulation disorders among children with a history of trauma: A frame of reference for speech-language pathologists. Language, Speech, and Hearing Services in Schools, 38, 109116.

Carr, J. L., Agnihotri, S., \& Keightley, M. (2010). Sensory processing and adaptive behavior deficits of children across the fetal alcohol spectrum disorder continuum. Alcoholism, Clinical and Experimental Research, 34, 1022-1032.

Chudley, A. E. (2011). Genetic factors contributing to FASD. In E. P. Riley, S. K. Clarren, J. Weinberg, \& E. Jonsson (Eds.), Fetal Alcohol Spectrum Disorder (pp. 109-126). Weinheim, Germany: Wiley-Blackwell.

Clarren, S. K. (1986). Neuropathology in fetal alcohol syndrome. In J. R. West (Ed.), Alcohol and brain development (pp. 158-166). New York, NY: Oxford University Press.

Fryer, S. L., McGee, C. L., Matt, G. E., Riley, E. P., \& Mattson, S. N. (2007). Evaluation of psychopathological conditions in children with heavy prenatal alcohol exposure. Pediatrics, 119, e733e741.

Green, C. R., Mihic, A. M., Nikkel, S. M., Stade, B. C., Rasmussen, C., Munoz, D. P., \& Reynolds, J. N. (2009). Executive function deficits in children with fetal alcohol spectrum disorders (FASD) measured using the Cambridge Neuropsychological Tests Automated Battery (CANTAB). Journal of Child Psychology and Psychiatry, 50, 688-697.

Jacobson, S. W., Chiodo, L. M., Sokol, R. J., \& Jacobson, J. L. (2002). Validity of maternal report of prenatal alcohol, cocaine, and smoking in relation to neurobehavioral outcome. Pediatrics, 109, 815-825.

Kodituwakku, P. W. (2007). Defining the behavioral phenotype in children with fetal alcohol spectrum disorders: A review. Neuroscience and Biobehavioral Reviews, 31, 192-201.

Kodituwakku, P. W. (2009). Neurocognitive profile in children with fetal alcohol spectrum disorders. Developmental Disabilities Research Reviews, 15, 218-224.

Kully-Martens, K., Denys, K., Treit, S., Tamana, S., \& Rasmussen, C. (2012). A review of social skills deficits in individuals with fetal alcohol spectrum disorders and prenatal alcohol exposure: Profiles, mechanisms, and interventions. Alcoholism, Clinical and Experimental Research, 36, 568-576.

Lee, K. T., Mattson, S. N., \& Riley, E. P. (2004). Classifying children with heavy prenatal alcohol exposure using measures of attention. Journal of the International Neuropsychological Society, 10, 271277.

Malone, M., \& Koren, G. (2012). Alcohol-induced behavioural problems in fetal alcohol spectrum disorders versus confounding behavioural problems. Journal of Population Therapeutics and Clinical Pharmacology, 19, e32-40.

Mattson, S. N., Roesch, S. C., Fagerlund, A., Autti-Ramo, I., Jones, K. L., May, P. A., . . . Riley, E. P. (2010). Toward a neurobehavioral profile of fetal alcohol spectrum disorders. Alcoholism, Clinical and Experimental Research, 34, 1640-1650.

McGee, C. L., Schonfeld, A. M., Roebuck-Spencer, T. M., Riley, E. P., \& Mattson, S. N. (2008). Children with heavy prenatal alcohol exposure demonstrate deficits on multiple measures of concept formation. Alcoholism, Clinical and Experimental Research, 32, 1388-1397.

Michaelis, E. K. (1990). Fetal alcohol exposure: Cellular toxicity and molecular events involved in toxicity. Alcoholism, Clinical and Experimental Research, 14, 819-826.

Morrow-Tlucak, M., Ernhart, C. B., Sokol, R. J., Martier, S., \& Ager, J. (1989). Underreporting of alcohol use in pregnancy: Relationship to alcohol problem history. Alcoholism, Clinical and Experimental Research, 13, 399-401. 
Nash, K., Sheard, E., Rovet, J., \& Koren, G. (2008). Understanding fetal alcohol spectrum disorders (FASDs): Toward identification of a behavioral phenotype. Scientific World Journal, 8, 873-882.

Nash, K., Stevens, S., Rovet, J., Fantus, E., Nulman, I., Sorbara, D., \& Koren, G. (2013). Towards identifying a characteristic neuropsychological profile for fetal alcohol spectrum disorders. 1. Analysis of the Motherisk FASD clinic. Journal of Population Therapeutics and Clinical Pharmacology, 20, e44-52.

Paintner, A., Williams, A. D., \& Burd, L. (2012a). Fetal alcohol spectrum disorders- implications for child neurology, Part 1: Prenatal exposure and dosimetry. Journal of Child Neurology, 27, 258-263.

Paintner, A., Williams, A. D., \& Burd, L. (2012b). Fetal alcohol spectrum disorders-implications for child neurology, Part 2: Diagnosis and management. Journal of Child Neurology, 27, 355-362.

Pei, J., Denys, K., Hughes, J., \& Rasmussen, C. (2011). Mental health issues in fetal alcohol spectrum disorder. Journal of Mental Health, 20, 438-448.

Pei, J., Job, J., Kully-Martens, K., \& Rasmussen, C. (2011). Executive function and memory in children with Fetal Alcohol Spectrum Disorder. Child Neuropsychology, 17, 290-309.

Quattlebaum, J. L., \& O'Connor, M. J. (2012). Higher functioning children with prenatal alcohol exposure: Is there a specific neurocognitive profile? Child Neuropsychology. 19, 561-578.

Riley, E. P., Infante, M. A., \& Warren, K. R. (2011). Fetal alcohol spectrum disorders: An overview. Neuropsychology Review, 21, 73-80.

Sulik, K. K., Johnston, M. C., \& Webb, M. A. (1981). Fetal alcohol syndrome: Embryogenesis in a mouse model. Science, 214, 936-938.

Courtney R. Green, PhD

Canada Fetal Alcohol Spectrum Disorder Research Network

Jocelynn L. Cook, PhD, MBA

Executive Director

Canada Fetal Alcohol Spectrum Disorder Research Network

1370 Potter Drive

Manotick, Ontario

K4M 1C3

Telephone: 613-220-0228

Fax: 613-701-0324

E-mail: jocelynn.cook@canfasd.ca 